\title{
XFEM Simulation of Pore-Induced Fracture of a Heterogeneous Concrete Beam in Three-Point Bending
}

\author{
C. C. Zhang, ${ }^{a}$ X. H. Yang, ${ }^{a, b, 1}$ and H. Gao ${ }^{a}$ \\ ${ }^{a}$ School of Civil Engineering and Mechanics, Huazhong University of Science and Technology, \\ Wuhan, China \\ ${ }^{\mathrm{b}}$ Hubei Key Laboratory of Engineering Structural Analysis and Safety Assessment, Wuhan, China \\ ${ }^{1}$ yangxinh@hust.edu.cn
}

The extended finite element method with the linear softening law is employed to simulate poreinduced crack initiation and propagation in heterogeneous plain concrete beams in three-point bending. A series of numerical simulations was performed and experimentally validated. The crack was found to always initiate at the beam bottom in the point nearest to the pore, propagating through it. When the pore has a larger offset from the beam midspan, the beam displays higher fracture resistance and energy dissipation spent for fracture. With an increase in a distance from the beam bottom, the ultimate load also increases, but the energy dissipation slightly varies. The pore sizes have a little effect on the fracture resistance of the concrete beam.

Keywords: heterogeneous plain concrete beam, three-point bending, extended finite element method, pore-induced fracture.

Introduction. As a complex multiphase inhomogeneous material, concrete consists of mortar, randomly distributed coarse aggregate, interface and various natural defects such as pores. To further understand its complicated failure mechanisms like micro-cracking, crack-branching, and tortuosity of the crack path, many experimental and numerical investigations were recently carried out [1-6].

In the experimental investigation, some non-destructive techniques such as acoustic emission (AE) and X-ray Computed Tomography (XCT) are usually used for monitoring the fracture process of concrete. For example, Goszczyńska [1] identified in which phase a crack is most likely to occur by comparing the AE signals. By the AE technique, Ohno and Ohtsu [7] classified the crack modes into three types: tensile, shear and mixed modes, occurring in the four-point bending concrete beam. Yang et al. [8] studied the failure evolution process of compressed concrete specimens with the aid of the XCT technique. These techniques are beneficial in analyzing concrete fracture while they are too expensive or time-consuming to conduct a mass of researches.

In the numerical investigation, it is essential to create a reasonable model because of complex and heterogeneous microstructures of concrete. The model should be stochastic and heterogeneous considering multiple phases and their interactions of concrete. At presenttime, both the digital image based (DIB) approach and the parameterization modeling (PM) approach are the most popular. The DIB approach can model multiphase concrete with real aggregate size, shape and distributions [9-11], but it will be costly or time consuming when a large number of specimens need to be analyzed. In the PM approach, indirect and direct algorithms are usually utilized to create concrete models with randomly distributed aggregates. In the indirect algorithms, spatially-varying random fields are generated and allocated to conventional finite element (FE) meshes [12], or aggregate and mortar phases are randomly assigned to lattice elements in lattice models $[10,13]$, to model the properties of different phases. It is easy to create a large number of models and thus fit for statistical analyses. In the direct algorithms, various phases with specific 
material properties, such as aggregates, mortar, interface and so on, are explicitly generated in a model $[14,15]$. Using the direct PM approach, some important parameters including the volume fractions, distribution, gradation, the shape of aggregates, and strength of interfaces between aggregate and mortar can be taken into account, and their effects on the mechanical behaviors can also be evaluated. At present, some direct PM algorithms have been developed to model different shape aggregates. Among them modeling spherical aggregates [16] is naturally the simplest. Yang and his coworkers [17-20] proposed a high-efficiency random aggregate generation and packing (RAGP) algorithm to generate polygonal or polyhedral aggregates for $2 \mathrm{D}$ or $3 \mathrm{D}$ modeling.

Numerical methods could be utilized to simulate the fracture process of materials. The phase field method is successfully used to simulate the fracture of single crystals and polycrystals in micrometer [21-24]. However, in the fracture simulation of macroscopic quasi-brittle materials such as concrete, the cohesive zone model (CZM), which provides an effective way to describe the crack propagation, was widely used in recent years [12, 15]. However, because a crack can only propagate along the element boundaries, refined meshes are required to get an accurate crack path. From the partition of unity approach, the extended finite element method (XFEM) was firstly proposed by Belytschko and Black [25]. Compared to the conventional finite element method, some enrichment functions are introduced in the XFEM so that the fracture could step across continuous finite elements. The XFEM was frequently used to efficiently simulate the crack growth in homogeneous materials [26, 27]. Recently, the XFEM was used to solve some fracture problems of infrastructure materials $[28,29]$.

Pores are a type of common defects in concrete structures. They can weaken the material locally and induce crack initiation. A large number of pores could be found in mesoscale concrete XCT scanned images [10, 30]. In general, the tensile strength of concrete decreases as the pore fraction increases [11,31]. Accordingly, it is worth noting that the pore effect on concrete fracture cannot be neglected. In this paper, a series of numerical concrete beam models with a single pore and randomly distributed coarse aggregate are created by using the RAGP algorithm [17-20], and the XFEM is employed to simulate the fracture behavior of concrete. The influences of pore location and size on fracture are evaluated.

\section{Basic Method.}

1.1. Governing Equations. In a two-dimensional (2D) problem, the equilibrium differential equation in a domain $\Omega$ shown in Fig. 1 can be expressed as follows [32, 33]:

$$
\nabla \cdot \sigma+\mathbf{b}=0
$$

where $\nabla$ is the gradient operator, $\sigma$ is the Cauchy stress tensor, and $\mathbf{b}$ is the unit body force vector. In the displacement boundary $\Gamma_{u}$, the traction boundary $\Gamma_{t}$, and the crack boundary $\Gamma_{c}$, the following conditions should be satisfied:

$$
\begin{array}{cc}
\mathbf{u}=\overline{\mathbf{u}} & \text { on } \Gamma_{u}, \\
\sigma \cdot \widehat{\mathbf{n}}=\overline{\mathbf{t}} & \text { on } \Gamma_{t}, \\
\sigma \cdot \widehat{\mathbf{n}}=0 & \text { on } \Gamma_{c},
\end{array}
$$

where $\mathbf{u}$ is the displacement vector, $\overline{\mathbf{u}}$ is the displacement vector on $\Gamma_{u}, \widehat{\mathbf{n}}$ is the external normal unit vector, and $\overline{\mathbf{t}}$ is the traction vector on $\Gamma_{t}$.

In small strain and displacement conditions, the relation between strain and displacement can be written as 


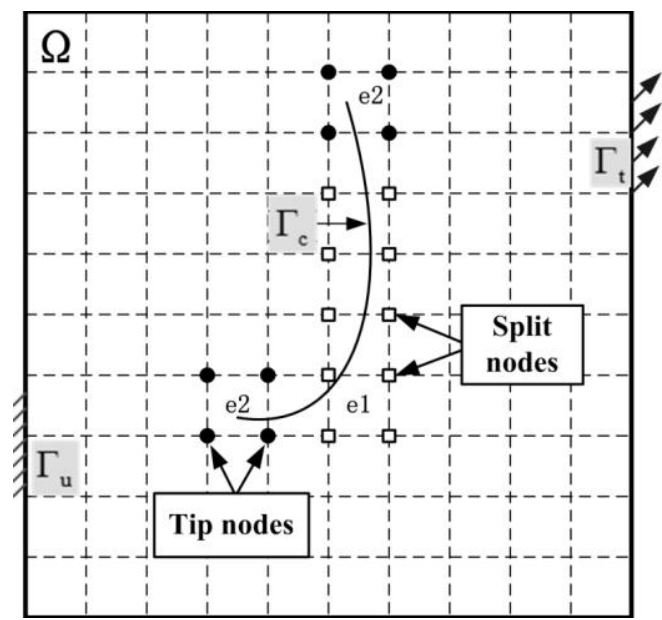

Fig. 1. Boundary conditions of a 2D domain with an internal crack and its typical discretization.

$$
\varepsilon=\nabla_{s} \mathbf{u}
$$

where $\nabla_{s}$ is the symmetric part of the gradient operator and $\varepsilon$ is the linear strain tensor.

For a linear elastic material, the constitutive relation can be given by

$$
\sigma=\mathbf{C}: \varepsilon
$$

where $\mathbf{C}$ is the elastic tensor.

The equilibrium equation in weak form can be given by [34]:

$$
\int_{\Omega} \sigma: \varepsilon d \Omega=\int_{\Omega} \mathbf{b} \cdot \mathbf{u} d \Omega+\int_{\Gamma_{t}} \overline{\mathbf{t}} \cdot \mathbf{u} d \Gamma
$$

1.2. XFEM Approximation for Cracks. The enriched trial and test displacement function for the XFEM is described in the matrix as follows [32]:

$$
\begin{aligned}
\mathbf{u}(\mathbf{X}) & =\sum_{i \in N^{s}} N_{i}(\mathbf{X}) \mathbf{u}_{i}+\sum_{j \in N^{c u t}} N_{j}(\mathbf{X})\left(H(\mathbf{X})-H\left(\mathbf{X}_{j}\right)\right) \mathbf{a}_{j}+ \\
& +\sum_{k \in N^{t i p}} N_{k}(\mathbf{X}) \sum_{\alpha=1}^{4}\left(\Psi_{\alpha}(\mathbf{X})-\Psi_{\alpha}\left(\mathbf{X}_{k}\right)\right) \mathbf{b}_{k}^{\alpha},
\end{aligned}
$$

where $\mathbf{X}$ is the arbitrary position matrix in the domain $\Omega$, while $\mathbf{X}_{j}$ and $\mathbf{X}_{k}$ are the position matrices at nodes $j$ and $k . N^{s}$ is the set of nodes in the discrete domain, $N^{\text {cut }}$ is the set of nodes, which belongs to these elements entirely cut by the crack, namely the split nodes marked as hollow squares in Fig. 1 , and $N^{\text {tip }}$ is the set of nodes which belongs to the crack-tip elements, namely the tip nodes marked as solid circles in Fig. 1. $N_{i}(\mathbf{X})$, $N_{j}(\mathbf{X})$, and $N_{k}(\mathbf{X})$ are the shape functions at nodes $i, j$, and $k$, respectively. $\mathbf{u}_{i}$ is the displacement matrix of node $i$, while $\mathbf{a}_{j}$ and $\mathbf{b}_{k}^{\alpha}$ are the accessional displacement matrices related to the crack face and crack-tip, respectively. $H(\mathbf{X})$ is the discontinuous enrichment function and can be expressed as 


$$
H(\mathbf{X})=\left\{\begin{array}{cl}
1 & \text { above the crack } \\
-1 & \text { below the crack }
\end{array}\right.
$$

The enrichment function of the crack-tip is usually determined by the displacement field near the crack-tip. For a homogeneous elastomer, $\Psi_{\alpha}(\mathbf{X})$ is given as follows:

$$
\left[\Psi_{1}, \Psi_{2}, \Psi_{3}, \Psi_{4}\right]=\left[\sqrt{r} \sin \frac{\theta}{2}, \sqrt{r} \cos \frac{\theta}{2}, \sqrt{r} \sin \frac{\theta}{2} \sin \theta, \sqrt{r} \cos \frac{\theta}{2} \sin \theta\right],
$$

where $r$ and $\theta$ are the local polar coordinates near the crack tip.

For a discretized model, the global equilibrium equation can be obtained by substituting Eq. (6) into Eq. (5)

$$
\mathbf{K d}=\mathbf{f},
$$

where $\mathbf{K}$ is the global stiffness matrix, $\mathbf{d}$ is the nodal displacement matrix, and $\mathbf{f}$ is the external force matrix. They can be given by assembling the element stiffness matrix $\mathbf{K}^{e}$, the nodal displacement matrix of the element $\mathbf{d}^{e}$, and the nodal force matrix of the element $\mathbf{f}^{e}$, respectively.

The element stiffness matrix with four nodes can be expressed as

$$
\mathbf{K}^{e}=\left[\begin{array}{lll}
\mathbf{K}^{u u} & \mathbf{K}^{u a} & \mathbf{K}^{u b} \\
\mathbf{K}^{a u} & \mathbf{K}^{a a} & \mathbf{K}^{a b} \\
\mathbf{K}^{b u} & \mathbf{K}^{b a} & \mathbf{K}^{b b}
\end{array}\right],
$$

where

$$
\mathbf{K}^{r s}=\int_{\Omega}\left(\mathbf{B}^{r}\right)^{T} \mathbf{C B}^{s} d \Omega \quad(r, s=u, a, b),
$$

with

$$
\begin{aligned}
\mathbf{B}^{u} & =\left[\begin{array}{llll}
\mathbf{B}_{1}^{u} & \mathbf{B}_{2}^{u} & \mathbf{B}_{3}^{u} & \mathbf{B}_{4}^{u}
\end{array}\right], \\
\mathbf{B}^{a} & =\left[\begin{array}{llll}
\mathbf{B}_{1}^{a} & \mathbf{B}_{2}^{a} & \mathbf{B}_{3}^{a} & \mathbf{B}_{4}^{a}
\end{array}\right], \\
\mathbf{B}^{b} & =\left[\begin{array}{llll}
\mathbf{B}^{b 1} & \mathbf{B}^{b 2} & \mathbf{B}^{b 3} & \mathbf{B}^{b 4}
\end{array}\right] .
\end{aligned}
$$

In the above three equations, we have

$$
\begin{gathered}
\mathbf{B}_{i}^{u}=\left[\begin{array}{cc}
\frac{\partial N_{i}}{\partial x} & 0 \\
0 & \frac{\partial N_{i}}{\partial y} \\
\frac{\partial N_{i}}{\partial y} & \frac{\partial N_{i}}{\partial x}
\end{array}\right], \quad i=1,2,3,4, \\
\mathbf{B}_{i}^{a}=\left(H(\mathbf{X})-H\left(\mathbf{X}_{i}\right)\right) \mathbf{B}_{i}^{u},
\end{gathered}
$$




$$
\mathbf{B}^{b \alpha}=\left[\begin{array}{llll}
\mathbf{B}_{1}^{b \alpha} & \mathbf{B}_{2}^{b \alpha} & \mathbf{B}_{3}^{b \alpha} & \mathbf{B}_{4}^{b \alpha}
\end{array}\right], \quad \alpha=1,2,3,4,
$$

with

$$
\mathbf{B}_{i}^{b \alpha}=\left(\Psi_{\alpha}(\mathbf{X})-\Psi_{\alpha}\left(\mathbf{X}_{i}\right)\right) \mathbf{B}_{i}^{u} .
$$

The nodal force matrix of the element can be expressed as

$$
\mathbf{f}^{e}=\left\{\begin{array}{llllll}
\mathbf{f}^{u} & \mathbf{f}^{a} & \mathbf{f}^{b 1} & \mathbf{f}^{b 2} & \mathbf{f}^{b 3} & \mathbf{f}^{b 4}
\end{array}\right\}^{T},
$$

where

$$
\begin{gathered}
\mathbf{f}^{u}=\left[\begin{array}{llll}
\mathbf{f}_{1}^{u} & \mathbf{f}_{2}^{u} & \mathbf{f}_{3}^{u} & \mathbf{f}_{4}^{u}
\end{array}\right], \\
\mathbf{f}^{a}=\left[\begin{array}{llll}
\mathbf{f}_{1}^{a} & \mathbf{f}_{2}^{a} & \mathbf{f}_{3}^{a} & \mathbf{f}_{4}^{a}
\end{array}\right], \\
\mathbf{f}^{b \alpha}=\left[\begin{array}{lllll}
\mathbf{f}_{1}^{b \alpha} & \mathbf{f}_{2}^{b \alpha} & \mathbf{f}_{3}^{b \alpha} & \mathbf{f}_{4}^{b \alpha}
\end{array}\right], \quad \alpha=1,2,3,4 .
\end{gathered}
$$

In the above three equations, we have

$$
\begin{gathered}
\mathbf{f}_{i}^{u}=\int_{\Omega} N_{i} \mathbf{b} d \Omega+\int_{\Gamma_{t}} N_{i} \overline{\mathbf{t}} d \Gamma \\
\mathbf{f}_{i}^{a}=\int_{\Omega}\left(H(\mathbf{X})-H\left(\mathbf{X}_{i}\right)\right) \mathbf{b} d \Omega+\int_{\Gamma_{t}} N_{i}\left(H(\mathbf{X})-H\left(\mathbf{X}_{i}\right)\right) \overline{\mathbf{t}} d \Gamma \\
\mathbf{f}_{i}^{b \alpha}=\int_{\Omega}\left(\Psi_{\alpha}(\mathbf{X})-\Psi_{\alpha}\left(\mathbf{X}_{i}\right)\right) \mathbf{b} d \Omega+\int_{\Gamma_{t}} N_{i}\left(\Psi_{\alpha}(\mathbf{X})-\Psi_{\alpha}\left(\mathbf{X}_{i}\right)\right) \overline{\mathbf{t}} d \Gamma .
\end{gathered}
$$

1.3. Level Set Method for Crack Propagation. In the XFEM, the level set method [35] is used to describe geometry evolution of crack surface and tip during crack growth iteration. The level set functions include the normal level set $\phi$ and the tangent one $\psi . \phi$ is a function of distance from the crack surface, while $\psi$ is a function of distance from the crack tip, as shown in Fig. 2. As the crack grows at each iterative step, both $\phi$ and $\psi$ are updated step by step. In this way, the crack growth is modeled.

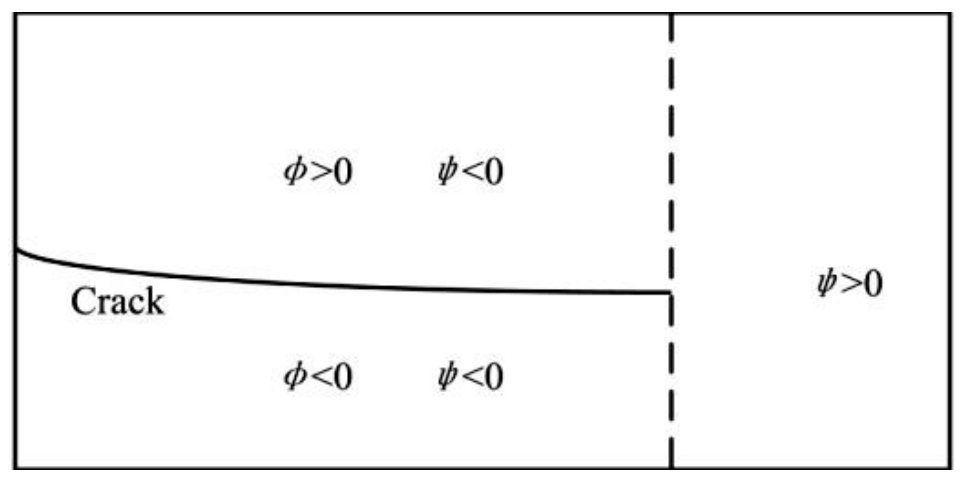

Fig. 2. Schematic of normal and tangent level set functions. 


\section{Experiments.}

2.1. Aggregate Size Distribution. A fuller grading curve suggests an ideal gradation of aggregates for concrete design. It can be written as a simple equation as follow [36]:

$$
P(d)=100\left(\frac{d}{d_{\max }}\right)^{n},
$$

where $P(d)$ is the cumulative percentage that aggregates pass a sieve with diameter $d$, $d_{\max }$ is the diameter of maximum coarse aggregate, and $n$ is a constant ranging between 0.45 and 0.70 .

Regarding the aperture diameter of a standard sieve, Eq. (20) can be discretized into several segments, then the volume of aggregates within the size range of $\left[d_{i}, d_{i+1}\right]$ can be obtained by [31]

$$
V_{\text {agg }}\left[d_{i}, d_{i+1}\right]=\frac{P\left(d_{i+1}\right)-P\left(d_{i}\right)}{P\left(d_{\max }\right)-P\left(d_{\min }\right)} R_{\text {agg }} V,
$$

where $d_{\min }$ is the minimum size of coarse aggregates, $R_{\text {agg }}$ is the volume fraction of coarse aggregates, and $V$ is the volume of concrete.

Before casting concrete, the coarse aggregates used in this paper are sieved. The passing percentages with the sieve size are listed in Table 1. The aggregates less than $4.75 \mathrm{~mm}$ are very few, and they are usually combined with the cement matrix as mortar [2] Accordingly, only the coarse aggregates with sizes greater than $4.75 \mathrm{~mm}$ are modeled in this paper. The coarse aggregates are discretized into five segments, and the corresponding five-segment gradation curve is shown in Fig. 3.

$\mathrm{T}$ a b 1 e 1

\section{Continuous Coarse Aggregate Gradation}

\begin{tabular}{|c|c|}
\hline Sieve size $(\mathrm{mm})$ & Total percentage passing (\%) \\
\hline 31.5 & 100.00 \\
\hline 26.5 & 92.36 \\
\hline 19.0 & 50.79 \\
\hline 16.0 & 34.01 \\
\hline 9.50 & 6.01 \\
\hline 4.75 & 0.32 \\
\hline
\end{tabular}

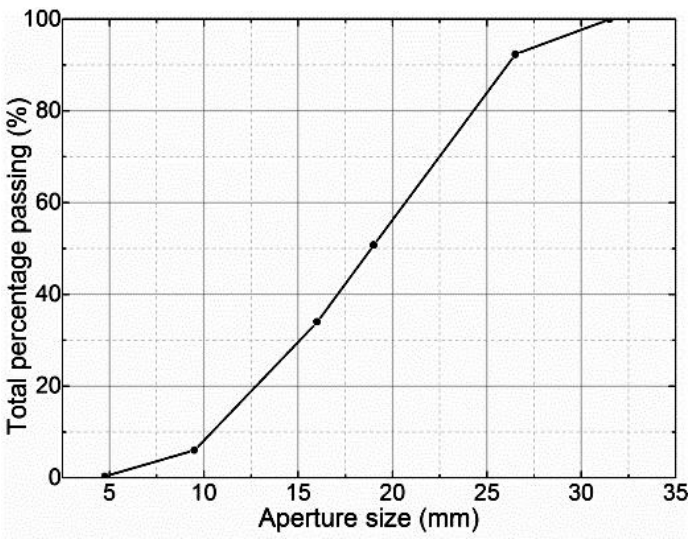

Fig. 3. Size distribution curve of aggregates with five grading segments. 
2.2. Three-Point Bending Test. Some concrete beam specimens with dimensions of $400 \times 100 \times 100 \mathrm{~mm}$ were prepared. Table 2 lists their ingredient contents in per cubic meter. In the present study, Ordinary Portland cement $\mathrm{P} \cdot \mathrm{O} 42.5$ (meeting the requirements of ASTM Type I) was used in all the specimens. The gradation of coarse aggregates is shown in Table 1. They were cured for seven days in a standard curing room. A $10 \mathrm{~mm}$ prefabricated through pore was located in the midspan or $30 \mathrm{~mm}$ off the midspan so that two groups of specimens labeled as L00U20D10 and L30U20D10 were prepared. The two values following $\mathrm{L}$ in the labels mean the offset of the pore from the beam midspan, the values following $U$ represents the distance of the pore from the beam bottom, and the values following $\mathrm{D}$ indicates the pore diameter. A sketch in Fig. 4 shows the boundary conditions of the beam and the pore location.

$\mathrm{T}$ a b 1 e 2

\section{Concrete Ingredients}

\begin{tabular}{|c|c|}
\hline Ingredients & Weight in per cubic meter of concrete $(\mathrm{kg})$ \\
\hline Cement & 454.2 \\
\hline Fine aggregate & 518.2 \\
\hline Coarse aggregate & 1079.4 \\
\hline Water & 241.5 \\
\hline
\end{tabular}

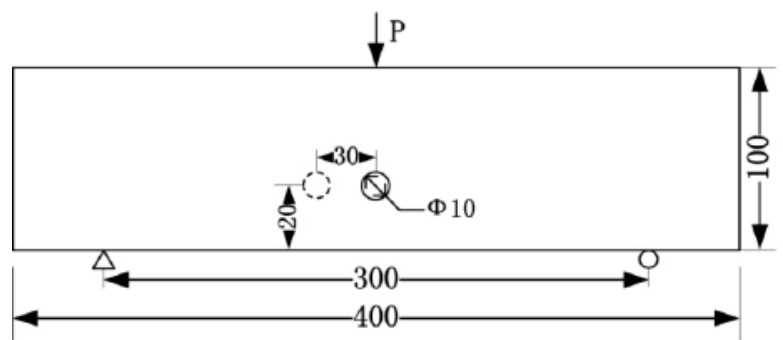

Fig. 4. Boundary conditions and pore location (unit: $\mathrm{mm}$ ).

The WDW-100E electronic universal testing machine was employed as shown in Fig. 5a and the corresponding data collection system is shown in Fig. 5b. There are two simple supports and a loading head in the three-point bending setup. The distance between the two supports is $300 \mathrm{~mm}$, and the loading head is located at the midspan of the beam. The YSS-C acoustic emission (AE) meter was employed to monitor the AE signal from the fractured concrete beams by a sensor attached to the center of the beam back. The experiments were performed at room temperature (about $20^{\circ} \mathrm{C}$ ), and displacement with a loading rate of $0.5 \mathrm{~mm} / \mathrm{min}$ was loaded. The fracture process was shot by the digital camera. Both the reactive force and the corresponding vertical displacement at the loading head were captured, and the AE event counts were also collected.

All the experiments were replicated three times. Their results are averaged to reduce the data randomness. The averaged load and AE event count vs. displacement curves are plotted in Fig. 6 for L00U20D10. The curves could be divided into three stages. In stage I, as the displacement increases from 0 to $0.356 \mathrm{~mm}$, the load nearly linearly increases to the ultimate load and the cumulative AE event count increases slowly. This indicates that the material behaves linearly elastic in stage I. In stage II, the cumulative AE event count increases sharply with increasing displacement, while the load decreases rapidly. When the displacement is greater than about $0.9 \mathrm{~mm}$, stage III comes, and the beam is completely fractured. In this stage, the load decreases gently, but the cumulative AE event count increases slowly. 


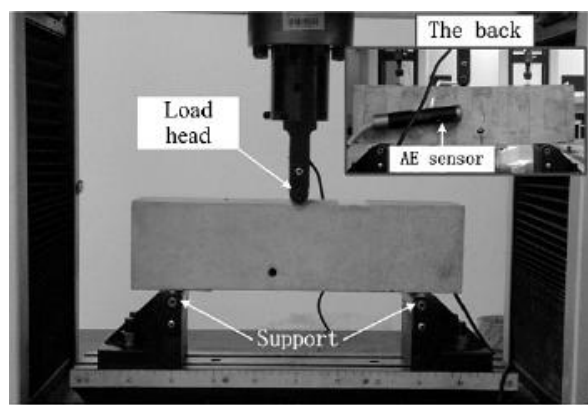

a

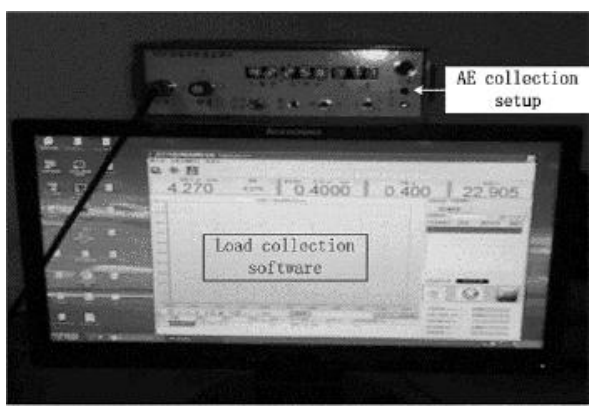

b

Fig. 5. Three-point bending setup (a) and data collection system (b).

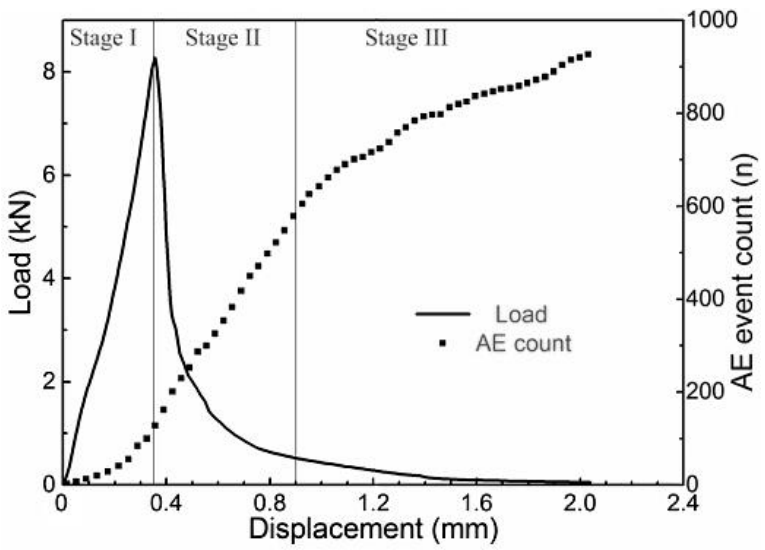

Fig. 6. The load and AE event count vs. displacement curves of L00U20D10.

\section{Numerical Simulations.}

3.1. Modeling Heterogeneous Concrete Beam. By using the RAGP algorithm [17-19], randomly distributed coarse aggregates with the gradation shown in Fig. 3 are generated and packed in the prescribed packing region of $400 \times 100 \mathrm{~mm}$. In the packing region, the part not occupied with the aggregates is treated as a homogeneous mortar matrix. A circular pore with a variable size and location is created. The whole model is assigned as the enriched region and discretized with the 4-node quadrilateral plane strain element. The element size is $2 \mathrm{~mm}$. The enriched function is added to the traditional finite element displacement function.

In this study, it is assumed that aggregates and cement paste are linear elastic before crack. The maximum principal stress criterion is used to judge if a crack will propagate when reaches the critical stress and the simplified linear softening law is employed herein. It is assumed that the shear parameters are the same as the normal ones because they are difficult to obtain by experiments. The material parameters used in this model, including the Young modulus $E$, Poisson's ratio $v$, the fracture energy $G_{f}$, and the critical fracture stress $t^{0}$ are listed in Table 3. These parameters are obtained from the experiments by the trial and error method.

Using the XFEM combined with Abaqus/CAE 6.14 commercial software, numerical three-point-bending tests were performed on the above heterogeneous concrete beam models. A displacement increasing from 0 to $2.4 \mathrm{~mm}$ was loaded at the top of beam midspan. Abaqus/Standard is utilized to solve the highly nonlinear equation systems. For convergence of solution, the computational time step is kept to be less than $0.0001 \mathrm{~s}$. 
$\mathrm{T}$ a b 1 e 3

Constitutive Model Parameters

\begin{tabular}{|c|c|c|c|c||}
\hline Material & $E, \mathrm{GPa}$ & $v$ & $G_{f}, \mathrm{~N} / \mathrm{m}$ & $t^{0}, \mathrm{MPa}$ \\
\hline Aggregate & 40 & 0.15 & 2000 & 8 \\
\hline Mortar matrix & 3 & 0.25 & 600 & 2 \\
\hline
\end{tabular}

3.2. Model Validation. To validate the numerical method, both the experimental and numerical load-displacement curves of L00U20D10 and L30U20D10, are plotted in Fig. 7. It is clear that there is a good correlation between the experimental and numerical curves although their slopes are slightly different before the ultimate load. Especially, both the experimental and numerical ultimate loads nearly correspond to the same displacement of about $0.35 \mathrm{~mm}$. This reflects that the XFEM method can well describe brittle failure and softening behaviors of concrete.

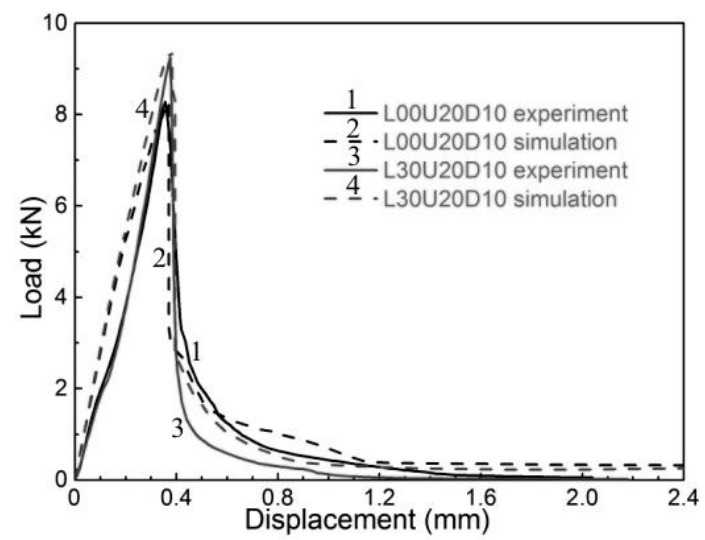

Fig. 7. Experimental and numerical load-displacement curves for different pore location beam.

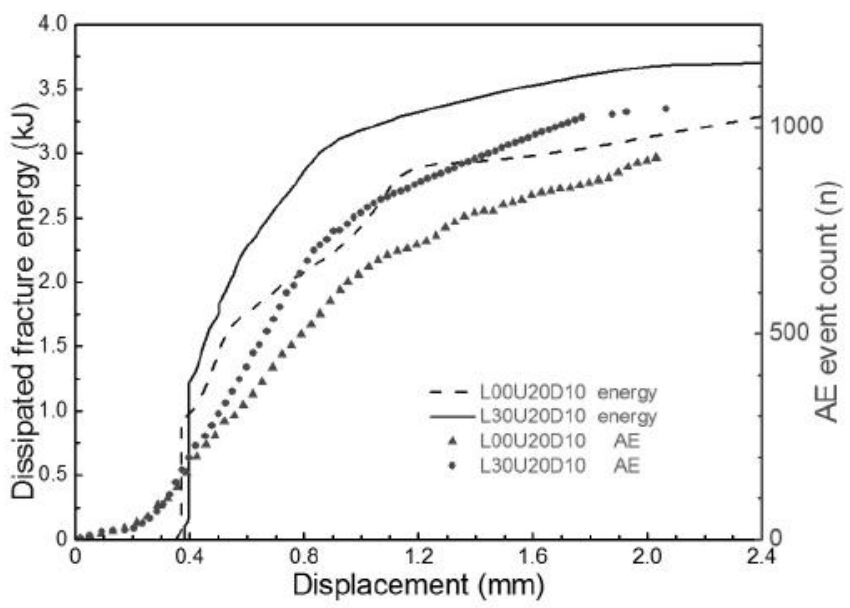

Fig. 8. Simulated dissipated fracture energies and experimental AE event counts for different pore location beams.

The simulated dissipated fracture energy is also qualitatively compared with the experimental AE event count. Both of them are plotted in Fig. 8. It can be found that they have a similar rising tendency and curve shape. When the displacement is less than $0.35 \mathrm{~mm}$, 
the dissipated fracture energy is 0 , and the AE event count increases slowly. However, when the displacement is greater than $0.35 \mathrm{~mm}$, they both begin to rise sharply with increasing the movement. When the displacement is greater than about $0.8 \mathrm{~mm}$, they both become gentle. Their good match further indicates that the present numerical method is viable.

\section{Analysis and Discussion.}

4.1. Crack Propagation and Field Evolution. As an example, Fig. 9 exhibits the numerical and triplicate experimental crack paths in L00U20D10 and L30U20D10. For easy identification, the tails of numerical crack paths are painted yellow. It is found that the numerical and experimental crack paths are very similar. Due to stress concentration induced by the pore, both the numerical and experimental crack paths pass through the pore and then walk toward the load point at the beam midspan. It indicates that the pore can intensively induce the beam to crack around it. This is possibly because the pore can locally weaken the fracture resistance of the beam. Besides, due to the uneven distribution of aggregates, all the predicted crack paths are tortuous. They are different from the cracks occurring in the homogeneous materials [26], but they are similar to the experimental paths in tortuosity. This reveals the considerable effect of internal microstructures on the crack propagation path.


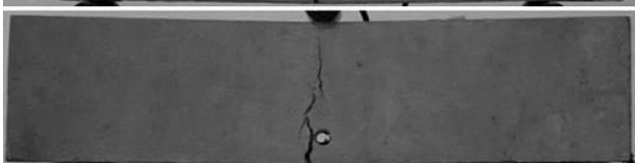

a

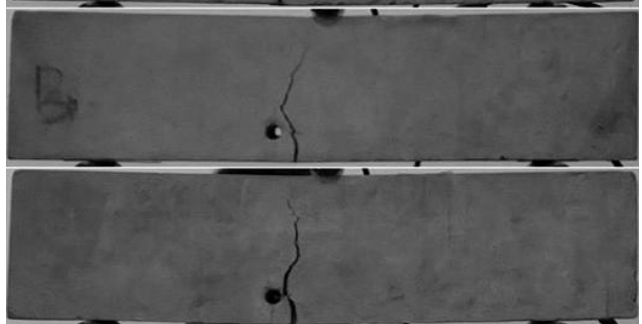

b

Fig. 9. Numerical and triplicate experimental crack paths of L00U20D10 (a) and L30U20D10 (b).

The transverse tensile stress distribution in different stages of the crack growth process of L00U20D10 is shown in Fig. 10. As the crack propagates, the stress concentration area ceaselessly moves forward. In stage I, the stress highly concentrates in the ligament between the crack tip and the pore before the crack is connected with the pore, as shown in Fig. 10a. When the crack exactly contacts the pore, in stage II, high stress concentration occurs at the top of the pore, as shown in Fig. 10b. After the crack leaves the pore behind, in stage III, the stress highly concentrates around the crack tip, as shown in Fig. 10c.

4.2. Pore Location Effect. The load-displacement and dissipated fracture energydisplacement curves of L00U20D10, L15U20D10 and L30U20D10 are plotted in Fig. 11a and $11 \mathrm{~b}$, respectively. Their offset from the midspan increases from 0,15 to $30 \mathrm{~mm}$ but both the distance from the bottom and the pore size remain unchanged, so the pore offset influence on fracture can be evaluated. It can be seen from Fig. 11 that both the ultimate load and failure dissipated fracture energy increases as the offset increases. It indicates that 


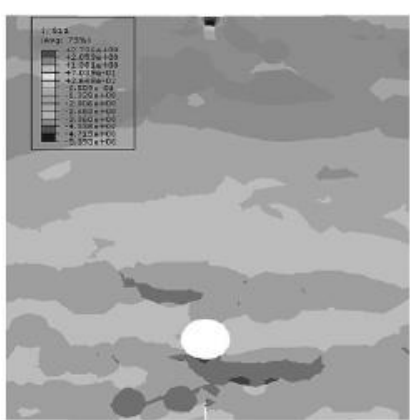

a

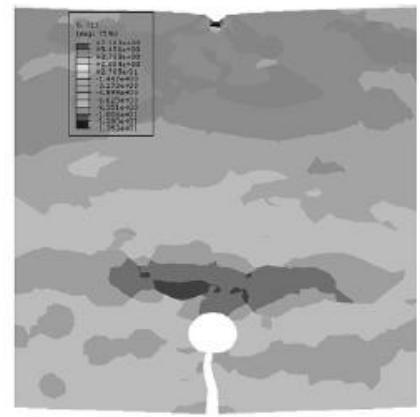

b

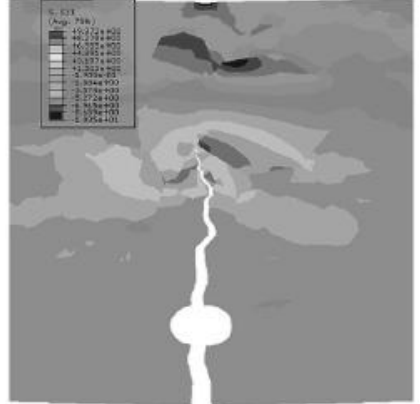

$\mathrm{c}$

Fig. 10. The transverse tensile stress distribution in different stages of the crack growth process of L00U20D10: (a) stage I; (b) stage II; (c) stage III.

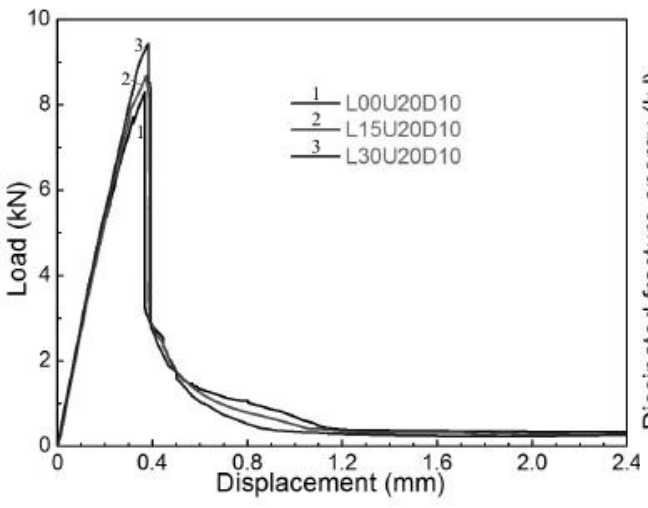

a

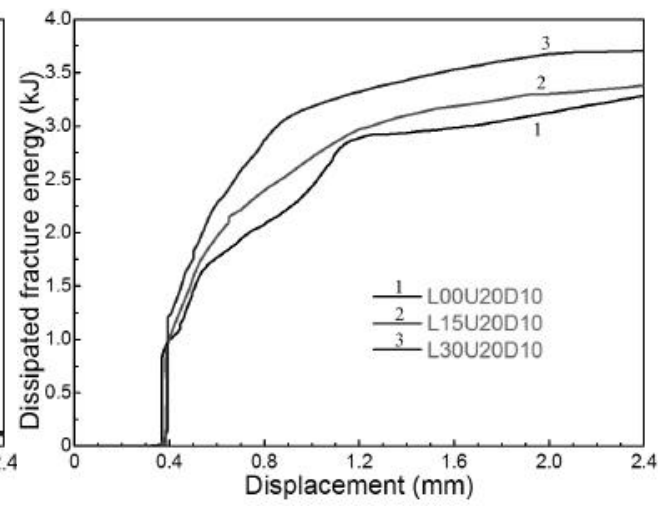

b

Fig. 11. Load and dissipated fracture energy vs. displacement curves for different pore offsets from the beam midspan.

the beam with a pore of greater offset has a higher fracture resistance and consumes more energy in the fracture process. This is easy to understand because an increasing pore offset would result in a reduced bending stress near the pore and a longer crack path.

To evaluate the effect of the pore distance from the beam bottom on the ultimate load, the fracture process of L00U10D10, L00U20D10, and L00U30D10 are simulated. Their ultimate load and failure dissipated fracture energy are plotted in Fig. 12a and b, respectively. It is seen that as the pore distance increases, the ultimate load increases. This indicates that the pore closer to the bottom could weaken the beam more badly. However, because they have almost the same crack path length, as shown in Fig. 9a, their failure dissipated fracture energy slightly varies.

4.3. Pore Size Effect. In general, the pore size has a significant impact on the fracture properties of the concrete beam. To evaluate this effect, the concrete beams of L00U20D8 and L00U20D12 are built. The ultimate load and failure dissipated fracture energy of L00U20D8, L00U20D10 and L00U20D12 are listed in Fig. 13. Apparently, as the pore size increases, both the fracture resistance and failure dissipated fracture energy slightly decreases. This is qualitatively consistent with the existing conclusion that the tensile strength of concrete decreases as the pore fraction increases [11,31]. Accordingly, the pore size has a little effect on the fracture resistance of the concrete beam. 


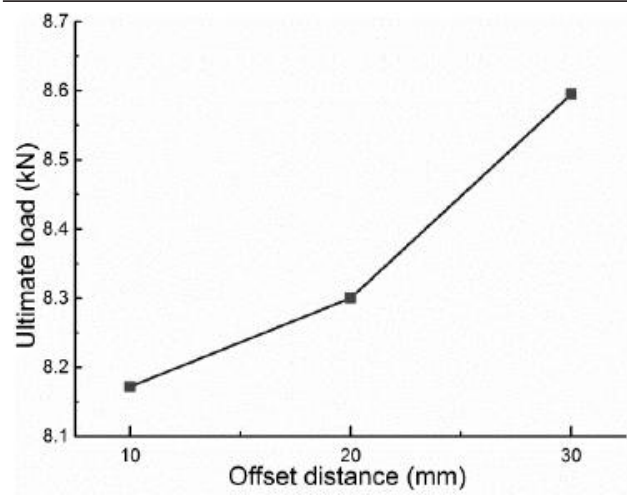

a

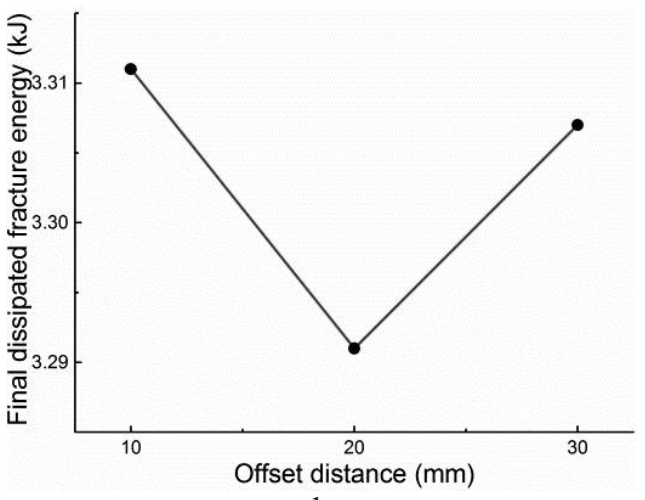

b

Fig. 12. Ultimate load and failure dissipated fracture energy for different pore distances from the beam bottom.

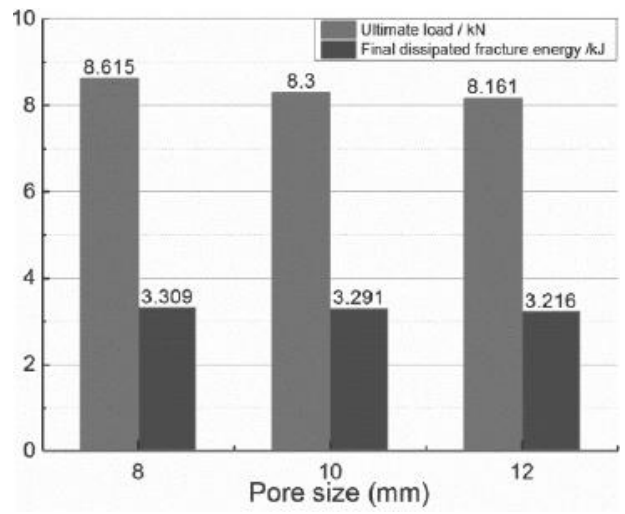

Fig. 13. The ultimate load and failure dissipated fracture energy for different pore size beams.

Conclusions. A series of 2D mesoscale heterogeneous concrete beam models are created by using the RAGP algorithm, and the XFEM is used to simulate the complicated pore-induced fracture processes under three-point bending. The following conclusions are obtained.

1. Due to stress concentration, the pore can locally weaken the fracture resistance of the beam. As a result, it is observed that the crack always initiates in the beam bottom the nearest to the pore and propagates through it.

2. Both the ultimate load and failure dissipated fracture energy increases as the pore offset from the beam midspan increases, so when the pore has a higher offset, the beam has a higher fracture resistance and dissipates more energy in the fracture process.

3. As the pore distance from the beam bottom increases, the ultimate load increases, so the pore closer to the bottom could weaken the beam more badly. But the failure dissipated fracture energy slightly varies with increasing pore distance.

4. As the pore size increases, both the fracture resistance and failure dissipated fracture energy slightly decreases, so the pore size has a little effect on the fracture resistance of the concrete beam.

Acknowledgments. This work is supported by the National Basic Research Program of China (973 Program: 2011CB013800).

1. B. Goszczyńska, "Analysis of the process of crack initiation and evolution in concrete with acoustic emission testing," Arch. Civ. Mech. Eng., 14, No. 1, 134-143 (2014). 
2. K. Ohno, K. Uji, A. Ueno, and M. Ohtsu, "Fracture process zone in notched concrete beam under three-point bending by acoustic emission," Constr. Build. Mater., 67, 139-145 (2014).

3. A. Ghosh and P. Chaudhuri, "Computational modeling of fracture in concrete using a meshfree meso-macro-multiscale method," Comp. Mater. Sci., 69, 204-215 (2013).

4. B. Rehder, K. Banh, and N. Neithalath, "Fracture behavior of pervious concretes: The effects of pore structure and fibers," Eng. Fract. Mech., 118, 1-16 (2014).

5. Y. Labadi and N. E. Hannachi, "Numerical simulation of brittle damage in concrete specimens," Strength Mater., 37, No. 3, 268-281 (2005).

6. H. Haeri, "Simulating the crack propagation mechanism of pre-cracked concrete specimens under shear loading conditions," Strength Mater., 47, No. 4, 618-632 (2015).

7. K. Ohno and M. Ohtsu, "Crack classification in concrete based on acoustic emission," Constr. Build. Mater., 24, No. 12, 2339-2346 (2010).

8. Z. Yang, W. Ren, M. Mostafavi, et al., "Characterisation of 3D fracture evolution in concrete using in-situ X-ray computed tomography testing and digital volume correlation," in: Proc. of the 8th Int. Conf. on Fracture Mechanics of Concrete and Concrete Structures (2013), pp. 1-7.

9. C. Başyiğit, B. Çomak, Ş. Kılınçarslan and I. Serkan Üncü, "Assessment of concrete compressive strength by image processing technique," Constr. Build. Mater., 37, 526-532 (2012).

10. A. P. Jivkov, D. L. Engelberg, R. Stein, and M. Petkovski, "Pore space and brittle damage evolution in concrete," Eng. Fract. Mech., 110, 378-395 (2013).

11. W. Ren, Z. Yang, R. Sharma, et al., "Two-dimensional X-ray CT image based meso-scale fracture modelling of concrete," Eng. Fract. Mech., 133, 24-39 (2015).

12. Z. J. Yang, X. T. Su, J. F. Chen, and G. H. Liu, "Monte Carlo simulation of complex cohesive fracture in random heterogeneous quasi-brittle materials," Int. J. Solids Struct., 46, No. 17, 3222-3234 (2009).

13. J. P. B. Leite, V. Slowik, and H. Mihashi, "Computer simulation of fracture processes of concrete using mesolevel models of lattice structures," Cement Concrete Res., 34, No. 6, 1025-1033 (2004).

14. C. M. López, I. Carol, and A. Aguado, "Meso-structural study of concrete fracture using interface elements. II: compression, biaxial and Brazilian test," Mater. Struct., 41, No. 3, 601-620 (2008).

15. A. Yin, X. Yang, G. Zeng, and H. Gao, "Fracture simulation of pre-cracked heterogeneous asphalt mixture beam with movable three-point bending load," Constr. Build. Mater., 65, 232-242 (2014).

16. G. Fu and W. Dekelbab, "3-D random packing of polydisperse particles and concrete aggregate grading," Powder Technol., 133, Nos. 1-3, 147-155 (2003).

17. A. Yin, X. Yang, S. Yang, and W. Jiang, "Multiscale fracture simulation of three-point bending asphalt mixture beam considering material heterogeneity," Eng. Fract Mech., 78, No. 12, 2414-2428 (2011).

18. S. F. Yang, X. H. Yang, A. Y. Yin and W. Jiang, "Three-dimensional numerical evaluation of influence factors of mechanical properties of asphalt mixture," J. Mech., 28, No. 3, 569-578 (2012).

19. A. Yin, X. Yang, C. Zhang, et al., "Three-dimensional heterogeneous fracture simulation of asphalt mixture under uniaxial tension with cohesive crack model," Constr. Build. Mater., 76, 103-117 (2015). 
20. C. Zhang, X. Yang, H. Gao, and H. Zhu, "Heterogeneous fracture simulation of three-point bending plain-concrete beam with double notches," Acta Mech. Solida Sin., 29, No. 3, 232-244 (2016).

21. J. D. Clayton and J. Knap, "Phase field modeling of directional fracture in anisotropic polycrystals," Comp. Mater. Sci., 98, 158-169 (2015).

22. J. D. Clayton and J. Knap, "Phase field modeling and simulation of coupled fracture and twinning in single crystals and polycrystals," Comput. Method. Appl. M., 312, 447-467 (2016).

23. C. Miehe, F. Welschinger and M. Hofacker, "Thermodynamically consistent phasefield models of fracture: Variational principles and multi-field FE implementations," Int. J. Numer. Meth. Eng., 83, No. 10, 1273-1311 (2010).

24. M. J. Borden, C. V. Verhoosel, M. A. Scott, et al., "A phase-field description of dynamic brittle fracture," Comput. Method. Appl. M., 217-220, 77-95 (2012).

25. T. Belytschko and T. Black, "Elastic crack growth in finite elements with minimal remeshing," Int. J. Numer. Meth. Eng., 45, No. 5, 601-620 (1999).

26. I. M. Lancaster, H. A. Khalid, and I. A. Kougioumtzoglou, "Extended FEM modelling of crack propagation using the semi-circular bending test," Constr. Build. Mater., 48, 270-277 (2013).

27. G. L. Golewski, P. Golewski, and T. Sadowski, "Numerical modelling crack propagation under Mode II fracture in plain concretes containing siliceous fly-ash additive using XFEM method," Comp. Mater. Sci., 62, 75-78 (2012).

28. N. Kenny and Q. Dai, "Investigation of fracture behavior of heterogeneous infrastructure materials with extended-finite-element method and image analysis," $J$. Mater. Civil. Eng., 23, No. 12, 1662-1671 (2011).

29. H. Wang, C. Zhang, L. Yang, and Z. You, "Study on the rubber-modified asphalt mixtures' cracking propagation using the extended finite element method," Constr. Build. Mater., 47, 223-230 (2013).

30. E. Piotrowska, Y. Malecot, and Y. Ke, "Experimental investigation of the effect of coarse aggregate shape and composition on concrete triaxial behavior," Mech. Mater., 79, 45-57 (2014).

31. X. F. Wang, Z. J. Yang, J. R. Yates, et al., "Monte Carlo simulations of mesoscale fracture modelling of concrete with random aggregates and pores," Constr. Build. Mater., 75, 35-45 (2015).

32. I. V. Singh, B. K. Mishra, S. Bhattacharya, and R. U. Patil, "The numerical simulation of fatigue crack growth using extended finite element method," Int. J. Fatigue, 36, No. 1, 109-119 (2012).

33. S. Kumar, I. V. Singh, B. K. Mishra, and T. Rabczuk, "Modeling and simulation of kinked cracks by virtual node XFEM," Comput. Method. Appl. M., 283, 1425-1466 (2015).

34. N. Moes, J. Dolbow, and T. Belytschko, "A finite element method for crack growth without remeshing," Int. J. Numer. Meth. Eng., 46, No. 1, 131-150 (1999).

35. M. Duflot, "A study of the representation of cracks with level sets," Int. J. Numer. Meth. Eng., 70, No. 11, 1261-1302 (2007).

36. M. Mungule and B. K. Raghuprasad, "Meso-scale studies in fracture of concrete: A numerical simulation," Comput. Struct., 89, Nos. 11-12, 912-920 (2011). 